\title{
Incidence of anal fistula after pyogenic perianal abscess drainage in Kingdom of Bahrain
}

\author{
Zahra Abdulla Isa Yusuf Hasan, Bayan Mohamed, Rawaa AlSayegh, Raed AlMarzooq \\ Department of Surgery, Salmaniya Medical Complex, Kingdom of Bahrain
}

Purpose: Perianal fistula is one of the most common anorectal diseases in adult patients, especially men. A relationship between pyogenic perianal abscess and fistula formation is established in multiple domains. This is the first exploration of such association among patients in the country as no related study has been published in Bahrain. We expect this study to be a foundation for future protocols and evidence-based practice.

Methods: A retrospective study was conducted in Salmaniya Medical Complex of Bahrain. A total of 109 patients with a diagnosis of anal abscess were included between 2015 and 2018. Data were collected from the electronic files database used in Salmaniya Medical Complex (iSeha) as well as phone calls to the patients. Collected data were analyzed using statistical software.

Results: The most predominant presentation of perianal abscess was pain. Over $50 \%$ of abscesses were classified as perianal (56.9\%) and among those, left-sided abscesses were more common, followed by right-, posterior-, and anterior-sited, respectively. No recurrence of abscess was recorded among $80 \%$ of patients. A fistula developed following abscess drainage in $33.9 \%$ of patients. Most fistulas (37.8\%) were diagnosed within 6 months or less from abscess drainage. Posterior fistulas were the most common, followed by anterior and left-sided fistulas.

Conclusion: The incidence of anal fistula in Bahrain after perianal abscess was 33.9\%. Most of the patients who developed a fistula following pyogenic abscess drainage were males and above the age of 40 years. The most common site for fistula was posterior.

\section{Keywords: Abscess; Recurrence; Drainage; Rectal fistula; General surgery}

\section{INTRODUCTION}

Perianal fistula is one of the most common anorectal diseases in adult patients, especially males [1]. A relationship between pyogenic perianal abscess and fistula formation is established in multiple domains, including etiology, anatomy, and pathophysiology [1]. In fact, both perianal abscesses and anal fistulas are considered to represent the acute and chronic manifestations, respec-

Received: Nov 9, 2020 - Revised: Jun 14, 2021 - Accepted: Jun 16, 2021 Correspondence to: Zahra Abdulla Isa Yusuf Hasan, MB, BCH, BAO (NUI,RCSI) LRCP \& SI.RCSI'18

Salmaniya Medical Complex, Rd No 2904, Manama, Kingdom of Bahrain Email: Zahraaabdulla1994@gmail.com

ORCID: https://orcid.org/0000-0002-0168-6409

(C) 2023 The Korean Society of Coloproctology

This is an open-access article distributed under the terms of the Creative Commons Attribution NonCommercial License (https://creativecommons.org/licenses/by-nc/4.0) which permits unrestricted noncommercial use, distribution, and reproduction in any medium, provided the original work is properly cited. tively, of the disease process of an infected anal gland [2].

The majority of fistula arise on the background of a preexisting abscess [3]. A study published in 2010 showed that the incidence of fistula-in-ano following the incision and drainage (I\&D) of perianal abscess was $31 \%$, with certain patient-related factors showing higher association rates with incidence [4].

The preferred management of perianal abscess, even in the occurrence of spontaneous discharge, is almost always surgical drainage which leads to an open cavity that takes about 3 to 4 weeks to heal. It is believed that an unsuccessful healing process may be an indicator of an underlying fistula. One study suggested that a fistula can be found in a third of the patients with an abscess, either at the time of abscess drainage or after it [3].

This research was conducted in Salmaniya Medical Complex in the Kingdom of Bahrain to explore the incidence of anal fistula after the drainage of pyogenic perianal abscess and investigate the factors related to the incidence of developing anal fistula after the drainage. This is the first exploration of such association among 
patients in the country as no study has been published on this topic in Bahrain. We expect this study to be a foundation to establish necessary guidelines and local protocols, as well as knowing the local incidence and statistics.

\section{METHODS}

\section{Ethical statements}

The ethics approval for this study was obtained from the Research Ethics Committee (REC) of Salmaniya Medical Complex. Informed consent was omitted by REC's approval.

\section{Setting}

Salmaniya Medical Complex opened in 1979 and has a capacity of nearly 1,200 beds. It is the first full-service public hospital in the Kingdom of Bahrain, with the first (and until 2018, the only) oncology center, receiving a total of 900 to 1,000 patients per day.

\section{Design and sampling}

A retrospective cohort study with the aim of finding the incidence of anal fistula following anal abscess drainage. From the admission office, a list of all patients above 18 years of age who had anal diseases between the period of 2015 to 2018 was obtained. The list included a total of 507 patients. Patients with diagnosed Crohn disease, diagnosed tuberculosis, known malignancy, previous fistula, and a diagnosis other than anal abscess (like anal fissure) were excluded.

\section{Data collection and analysis}

The parameters of the study were collected from the electronic files database system used in Salmaniya Medical Complex (iSeha) and through phone calls. Extracted information was documented in a designated form and included; age at onset, duration until diagnosis, duration until drainage, type of treatment, abscess site, number of abscess, number of recurrence, associated symptoms, antibiotic use, culture, and fistula formation and location. Using a telephone questionnaire, patients were asked to classify themselves into their appropriate socioeconomic status based on their average monthly income (poor, middle, or high) which is based on the patient's own classification. In addition, they were asked about the nature of their job (office vs. field job) and hence were grouped into an active and non-active occupation. The form was electronically converted and kept in a password-protected file until the end of the data collection phase and was only accessible to the researchers. Data were then entered into the IBM SPSS ver. 25.0 (IBM Corp) for analysis. Descriptive statistics were generated. A P-value of $<0.05$ was considered to be statistically significant.

\section{RESULTS}

Upon reviewing the initial list of 507 patients and applying the exclusion criteria, patients with insufficient data were eliminated. As
Table 1. Sample demographics

\begin{tabular}{|c|c|}
\hline Variable & Data \\
\hline No. of patients & 109 \\
\hline \multicolumn{2}{|c|}{ Age at diagnosis (yr) } \\
\hline$\leq 40$ & $46(42.2)$ \\
\hline$>40$ & $63(57.8)$ \\
\hline \multicolumn{2}{|l|}{ Sex } \\
\hline Male & $89(81.7)$ \\
\hline Female & $20(18.3)$ \\
\hline \multicolumn{2}{|l|}{ Nationality } \\
\hline Bahraini & $79(72.5)$ \\
\hline Non-Bahraini & $30(27.5)$ \\
\hline \multicolumn{2}{|l|}{ Marital status } \\
\hline Married & $39(35.8)$ \\
\hline Single & $11(10.1)$ \\
\hline Unknown & $59(54.1)$ \\
\hline \multicolumn{2}{|l|}{ Occupation } \\
\hline Active & $15(13.8)$ \\
\hline Non-active & $27(24.8)$ \\
\hline Unemployed & $6(5.5)$ \\
\hline Unknown & $61(56.0)$ \\
\hline \multicolumn{2}{|c|}{ Socioeconomic status } \\
\hline Poor & $16(14.7)$ \\
\hline Middle & $27(24.8)$ \\
\hline High & $6(5.5)$ \\
\hline Unknown & $60(55.0)$ \\
\hline \multicolumn{2}{|c|}{ Immunocompromised } \\
\hline Yes, diabetes & $40(36.7)$ \\
\hline Yes, others & $1(0.92)$ \\
\hline No & $29(26.6)$ \\
\hline Unknown & $37(33.9)$ \\
\hline \multicolumn{2}{|l|}{ Smoking } \\
\hline Yes & $32(29.4)$ \\
\hline No & $20(18.3)$ \\
\hline Unknown & $57(52.3)$ \\
\hline \multicolumn{2}{|c|}{ Alcohol consumption } \\
\hline Yes & $9(8.3)$ \\
\hline No & $42(38.5)$ \\
\hline Unknown & $58(53.2)$ \\
\hline \multicolumn{2}{|l|}{ Abscess site } \\
\hline Perianal & $62(56.9)$ \\
\hline Left & $23(21.1)$ \\
\hline Right & $15(13.8)$ \\
\hline Posterior & $14(12.8)$ \\
\hline Anterior & $10(9.2)$ \\
\hline Intersphincteric & $3(2.8)$ \\
\hline Unknown & $44(40.3)$ \\
\hline
\end{tabular}

Values are presented as number (\%). 
Table 2. Characteristic of patients who developed fistula vs. patients who didn't after perianal abscess drainage

\begin{tabular}{|c|c|c|c|c|}
\hline Characteristic & Fistula & No-fistula & 0dds ratio & P-value \\
\hline Sex & & & 0.9443 & 0.553 \\
\hline Male & 30 & 59 & & \\
\hline Female & 7 & 13 & & \\
\hline Nationality & & & 0.5486 & 0.058 \\
\hline Bahraini & 33 & 46 & & \\
\hline Non-Bahraini & 34 & 26 & & \\
\hline Marital status & & & & 0.002 \\
\hline Married & 19 & 20 & & \\
\hline Single & 0 & 11 & & \\
\hline Unknown & 18 & 41 & & \\
\hline Job activity level & & & 0.9697 & 0.613 \\
\hline Active & 6 & 9 & & \\
\hline Not active & 11 & 16 & & \\
\hline Not working & 1 & 5 & & \\
\hline Uknown & 19 & 42 & & \\
\hline Socioeconomic status & & & & 0.031 \\
\hline Poor & 7 & 9 & & \\
\hline Middle & 9 & 17 & & \\
\hline High & 2 & 4 & & \\
\hline Unknown & 19 & 41 & & \\
\hline Smoking status & & & 0.7154 & 0.288 \\
\hline Smoker & 6 & 14 & & \\
\hline Non-smoker & 13 & 18 & & \\
\hline Unkown & 18 & 40 & & \\
\hline Alcohol consumption & & & 0.4034 & 0.247 \\
\hline Yes & 2 & 7 & & \\
\hline No & 17 & 24 & & \\
\hline Unkown & 18 & 41 & & \\
\hline
\end{tabular}

a result, a total of 109 patients were enrolled in the study. The mean age of the sample is 44.9 years, ranging from 20 to 75 years. More than half of the participants were older than 40 years at the time of the study. The majority of the patients were males, forming $81.7 \%$ of the study population compared to $18.3 \%$ for females. The mean age of abscess diagnosis for females was lower than that of their males' counterparts with it being 39 years for females as opposed to 43 years for males (Tables 1,2).

The most predominant presenting complaint of an abscess was pain, followed closely by a swelling noticed by the patient. More than half of the patients (57.8\%) were diagnosed within less than 1 month from symptoms onset while only $3.7 \%$ got their diagnosis after more than 6 months. All patients were treated with I\&D of the abscess except for one who underwent examination under anesthesia + suction ligation. Over $64.2 \%$ of the patients underwent I\&D of the abscess no more than 3 days from diagnosis. Nearly half of the patients (54 patients, $49.5 \%$ ) stayed at the hospital for 3 days or less following the I\&D, 40.4\% stayed for 4 to 7 days inclusive, $8.2 \%$ stayed for more than 7 days with the remaining 2 patients having an unknown length of stay. A follow-up appointment after 2 weeks was given for $65.1 \%$ of the patients.

When it comes to the site of the abscess, over half were classified as perianal (56.9\%) and among those, left-sided abscesses were more frequently occurring followed by right and posteriorly sited. Almost all of the patients had only one abscess at the time of diagnosis $(94.9 \%)$. Only $20.2 \%$ of the patients had 1 or more recurrences of the abscess while the remaining $79.8 \%$ did not experience any recurrence.

Following I\&D, 72.5\% of the patients received a course of antibiotics with metronidazole being the most commonly prescribed antibiotics. Only 2 patients from the studied sample underwent I\&D without a course of antibiotics prescribed after it. The patients of $36.7 \%$ received the antibiotics for more than 5 days. The preferred route of administration was oral (59.6\%). Out of the 62 available culture results, Escherichia coli was the most isolated organism and Staphylococcus aureus was the least commonly isolated microorganism (Table 3).

Out of the 109 patients included in the study, 37 of them (33.9\%) developed a fistula (chart 1). Of the recurrences, $86.3 \%$ occurred in males while $13.6 \%$ in females. Average follow-up time was around 22 months, ranging from a minimum of 1 month to a maximum of 60 months follow-up period. The patients of $37.8 \%$ were diagnosed with a fistula within 6 months or less from the abscess with only $20 \%$ of the fistulas occurring after more than 6 months. The patients of $16.2 \%$ had a coexisting fistula at the time of the diagnosis. A posteriorly located fistula developed in 54.1\% of the patients, followed by anteriorly located fistulas (18.9\%), left (8.1\%), and right (2.7\%) while $16.2 \%$ had no documentation of fistula position (Table 4). Socioeconomic status was significantly related to fistula development (Table 2).

\section{DISCUSSION}

An infected gland is proposed to be the first step of the pathophysiological pathway behind developing a perianal abscess acutely, and chronically a perianal fistula. Nevertheless, the reason why some perianal abscesses develop further to fistulas is not clear. The prevalence of anorectal abscess is believed to be an understatement as the majority of patients either fail to seek medical help or are given the diagnosis of symptomatic hemorrhoids [5]. I\&D is the most commonly adopted procedure for treating perianal abscesses $[3,6,7]$, after which, pain relief is usually immediate [5].

In the studied sample, most patients showed no sign of recurrence of the abscess and $66.1 \%$ did not develop an anal fistula. Those results are in agreement with the published literature that 
Table 3. Abscess microorganisms and antibiotics

\begin{tabular}{|c|c|}
\hline Variable & Data \\
\hline \multicolumn{2}{|l|}{ Antibiotic usage } \\
\hline Unknown & $28(25.7)$ \\
\hline Given & $79(72.5)$ \\
\hline Not given & $2(1.8)$ \\
\hline \multicolumn{2}{|l|}{ Antibiotic duration (day) } \\
\hline Unknown & $38(34.9)$ \\
\hline$\leq 5$ & $31(28.4)$ \\
\hline$>5$ & $40(36.7)$ \\
\hline \multicolumn{2}{|l|}{ Type of antibiotic } \\
\hline Metronidazole & $42(35.3)$ \\
\hline Ciprofloxacin & $39(32.8)$ \\
\hline Co-amoxiclav & $19(16.0)$ \\
\hline Cefuroxime & $9(7.6)$ \\
\hline Fusidic acid (topical) & $5(4.2)$ \\
\hline Clarithromycin & $2(1.7)$ \\
\hline Tazobactam & $2(1.7)$ \\
\hline Meropenem & $1(0.8)$ \\
\hline \multicolumn{2}{|l|}{ Route of antibiotic } \\
\hline Oral & $60(83.3)$ \\
\hline IV & $3(4.2)$ \\
\hline Topical & $1(1.4)$ \\
\hline IV + oral & $4(5.6)$ \\
\hline Topical + oral & $4(5.6)$ \\
\hline \multicolumn{2}{|l|}{ Abscess organism } \\
\hline Unknown & $42(38.5)$ \\
\hline Escherichia coli & $25(22.9)$ \\
\hline Mixed growth & $10(9.2)$ \\
\hline Sterile & $9(8.3)$ \\
\hline Klebsiella & $8(7.3)$ \\
\hline Others & $6(5.5)$ \\
\hline Not done & $5(4.6)$ \\
\hline Staphylococcus aureus & $4(3.7)$ \\
\hline
\end{tabular}

Values are presented as number (\%).

IV, intravenous.

states abscess recurrence will occur in only $3 \%$ to $44 \%$ of the patients while a fistula will develop in $26 \%$ to $50 \%$ of the patients following I\&D.

An association between the site of the abscess and the risk of recurrence $(\mathrm{P}=0.353)$ or developing a fistula $(\mathrm{P}=0.38)$ was not evident in our sample which is consistent with previous studies $[8,9]$. In addition, no link was found between the duration from diagnosis of the abscess until its drainage on the risk of recurrence
Table 4. Incidence of anal fistula in both males and females after incision and drainage of anorectal abscesses according to their location

\begin{tabular}{lccc}
\hline Location & Male $(\mathrm{n}=30)$ & Female $(\mathrm{n}=7)$ & Total $(\mathrm{n}=37)$ \\
\hline Anterior & $6(20.0)$ & $1(14.3)$ & $7(18.9)$ \\
Posterior & $16(53.3)$ & $4(57.1)$ & $20(54.1)$ \\
Right & $1(3.3)$ & $0(0)$ & $1(2.7)$ \\
Left & $2(6.7)$ & $1(14.3)$ & $3(8.1)$ \\
Unknown & $5(16.7)$ & $1(14.3)$ & $6(16.2)$ \\
\hline
\end{tabular}

Values are presented as number (\%).

$(\mathrm{P}=0.13)$ or recurrence of the abscess. A possible explanation for that could be the varying amount of time between patients from symptoms onset until the first presentation. While the majority of patients presented to the hospital within less than a month from symptoms onset, this was a self-reported history. Lack of public awareness regarding the condition, a perceived feeling of shame due to the conservative nature of the culture as well as the faulty diagnosis could all be possible factors that stop patients from seeking medical care earlier.

Among the patients who developed an anal fistula, the most common location was posterior. This could be attributed to the fact that anal glands are more numerous posteriorly [10,11]. Anal glands are also more numerous in males when compared to females, and this could be a reason why this disease process shows a sharp sex variation in its incidence [12].

Poor socioeconomic status is one of the main risk factors for developing anal fistula observed in many studies [13, 14]. In our study and among the patients who answered our telephone questionnaire about their socioeconomic status, we noticed that $88.8 \%$ of those who developed a fistula ranked their status to be between poor to middle. This may be attributed to poor hygiene and limited accessibility to treatment with fewer waiting hours which is not always possible in a public hospital.

Antibiotics were prescribed in over $70 \%$ of the patients in the studied sample. When it comes to prescribing antibiotics following I\&D of a pyogenic perianal abscess, the evidence is controversial. Multiple hypotheses exist. On the one hand, some believe that a course of antibiotics following drainage will reduce the odds of developing a fistula tract by eradicating residual infection especially in patients over 45 years [15]; but on the other hand, the results of a randomized trial reported opposing conclusions [16] which only highlights the need of a systematic review of the available literature in order to propose clear guidelines regarding the use of antibiotics following the drainage of a perianal abscess [17]. In this study, no association between the use of antibiotics and the risk of developing a fistula or a recurrence of the abscess was found. Additionally, a sterile culture was obtained in a number of patients who were also prescribed antibiotics. This is a critical point-unnecessary antibiotics prescriptions prophylactically and without parallel evidence of infection will increase the incidence 
of antimicrobial resistance, a serious problem considering the slowing development of new antibiotics [18]. According to the American Society of Colon and Rectal Surgeons, antibiotics have a limited role in treating uncomplicated anal abscesses and may only be considered in patients with significant cellulitis, those who have underlying immunosuppression or preoperatively in patients with valve pathology, in concurrence with the recommendations of the American Heart Association [19, 20]. A culture was done in all patients who underwent abscess drainage in the studied sample. While it would be beneficial before prescribing antibiotics, in atypical presentations or immunocompromised patients, to distinguish a crypto-glandular abscess from a skin abscess (colonic flora vs. staphylococcal species) or in patients at risk of having methicillin resistance Staphylococcus aureus, a routine culture in other contexts will not add to the treatment regimen and will instead be a waste of resources.

In conclusion, the incidence of perianal fistula following perianal abscess drainage was found to be $34 \%$. A higher incidence of fistula was found among male patients who are aged 40 years and above. The most common site of fistula was found to be posterior. Incidentally, antibiotics were found to be used in almost all patients. The practice of prescribing antibiotics following anal abscess drainage is not supported by currently available evidence. Furthermore, inconsistency of terms used in recording procedures' details and patients' information was noted. An audit is recommended for the practice and documentation of the management of perianal abscess.

\section{CONFLICT OF INTEREST}

No potential conflict of interest relevant to this article was reported.

\section{FUNDING}

None.

\section{REFERENCES}

1. Hasan RM. Incidence of fistula after management of perianal abscess. J Coloproctol (Rio de Janeiro) 2016;36:216-9.

2. Whiteford MH. Perianal abscess/fistula disease. Clin Colon Rectal Surg 2007;20:102-9.

3. Sahnan K, Adegbola SO, Tozer PJ, Watfah J, Phillips RK. Perianal abscess. BMJ 2017;356:j475.

4. Lohsiriwat V, Yodying H, Lohsiriwat D. Incidence and factors influencing the development of fistula-in-ano after incision and drainage of perianal abscesses. J Med Assoc Thai 2010;93:61-5.

5. Sigmon DF, Emmanuel B, Tuma F. Perianal abscess [Internet].
StatPearls Publishing; 2020 Jan [updated 2020 Feb 25; cited 2020 Mar 10]. Available from: https://www.ncbi.nlm.nih.gov/books/ NBK459167

6. Malik AI, Nelson RL, Tou S. Incision and drainage of perianal abscess with or without treatment of anal fistula. Cochrane Database Syst Rev 2010;(7):CD006827.

7. Hämäläinen KP, Sainio AP. Incidence of fistulas after drainage of acute anorectal abscesses. Dis Colon Rectum 1998;41:1357-62.

8. Akkapulu N, Dere Ö, Zaim G, Soy HE, Özmen T, Doğrul AB. A retrospective analysis of 93 cases with anorectal abscess in a rural state hospital. Ulus Cerrahi Derg 2014;31:5-8.

9. Yano T, Asano M, Matsuda Y, Kawakami K, Nakai K, Nonaka M. Prognostic factors for recurrence following the initial drainage of an anorectal abscess. Int J Colorectal Dis 2010;25:1495-8.

10. Abeysuriya V, Salgado LS, Samarasekera DN. The distribution of the anal glands and the variable regional occurrence of fistula-inano: is there a relationship? Tech Coloproctol 2010;14:317-21.

11. Seow-Choen F, Ho JM. Histoanatomy of anal glands. Dis Colon Rectum 1994;37:1215-8.

12. Lilius HG. Fistula-in-ano, an investigation of human foetal anal ducts and intramuscular glands and a clinical study of $150 \mathrm{pa}-$ tients. Acta Chir Scand Suppl 1968;383:7-88.

13. Ramteke S, Gupta AM, Soni P. Clinical study of fistula in ano. J Evid Based Med Healthc 2017;4:595-9.

14. Siddharth R, Kumar GA, Sreedhar S. Clinical study of fistula in ano. J Evol Med Dent Sci 2015;4:15082-8.

15. Nunoo-Mensah JW, Balasubramaniam S, Wasserberg N, Artinyan A, Gonzalez-Ruiz C, Kaiser AM, et al. Fistula-in-ano: do antibiotics make a difference? Int J Colorectal Dis 2006;21:441-3.

16. Sözener U, Gedik E, Kessaf Aslar A, Ergun H, Halil Elhan A, Memikoğlu O, et al. Does adjuvant antibiotic treatment after drainage of anorectal abscess prevent development of anal fistulas?: a randomized, placebo-controlled, double-blind, multicenter study. Dis Colon Rectum 2011;54:923-9.

17. Baker L, Williams L, Winter R, Cahill C, Davis A, Fergusson D. Influence of adjuvant antibiotics on fistula formation following incision and drainage of anorectal abscesses: a systematic review protocol. Syst Rev 2019;8:95.

18. Doron S, Davidson LE. Antimicrobial stewardship. Mayo Clin Proc 2011;86:1113-23.

19. Steele SR, Kumar R, Feingold DL, Rafferty JL, Buie WD; Standards Practice Task Force of the American Society of Colon and Rectal Surgeons. Practice parameters for the management of perianal abscess and fistula-in-ano. Dis Colon Rectum 2011;54: 1465-74.

20. Vogel JD, Johnson EK, Morris AM, Paquette IM, Saclarides TJ, Feingold DL, et al. Clinical practice guideline for the management of anorectal abscess, fistula-in-ano, and rectovaginal fistula. Dis Colon Rectum 2016;59:1117-33. 